\title{
A Novel Laser Guidance System for Alignment of Linear Surgical Tools: Its Principles and Performance Evaluation as a Man-Machine System
}

\author{
Toshihiko Sasama ${ }^{1}$, Nobuhiko Sugano ${ }^{2}$, Yoshinobu Sato ${ }^{1}$, Yasuyuki Momoi ${ }^{3}$, \\ Tsuyoshi Koyama ${ }^{2}$, Yoshikazu Nakajima ${ }^{1}$, Ichiro Sakuma ${ }^{4}$, Masakatsu Fujie ${ }^{5}$, \\ Kazuo Yonenobu ${ }^{6}$, Takahiro Ochi ${ }^{2}$, and Shinichi Tamura ${ }^{1}$ \\ 1 Division of Interdisciplinary Image Analysis, \\ Osaka University Graduate School of Medicine \\ 2 Department of Orthopaedic Surgery, Osaka University Graduate School of Medicine \\ 3 Mechanical Engineering Research Laboratory, Hitachi Ltd \\ 4 Department of Environmental Studies, Graduate School of Frontier Sciences, \\ The University of Tokyo \\ 5 Department of Mechanical Engineering, Waseda University \\ ${ }^{6}$ Department of Orthopaedic surgery, Osaka Minami National Hospital
}

\begin{abstract}
A novel laser guidance system that uses dual laser beam shooters for the alignment of linear surgical tools is presented. In the proposed system, the intersection of two laser planes generated by dual laser shooters placed at two fixed locations defines the straight insertion path of a surgical tool. The guidance information is directly projected onto the patient and the surgical tool. Our assumption is that a linear surgical tool has cylindrical shape or that a cylindrical sleeve is attached to the tool so that the sleeve and tool axes are aligned. The guidance procedure is formulated mainly using the property that the two laser planes are projected as two parallel straight lines onto the cylindrical tool surface if and only if the cylinder axis direction is the same as the direction of the intersection of the two laser planes. Unlike conventional augmented reality systems, the proposed system does not require the wearing of glasses or mirrors to be placed between the surgeon and patient. In our experiments, a surgeon used the system to align wires according to the alignment procedure, and the overall accuracy and alignment time were evaluated. The evaluations were considered not to be simply of a mechanical system but of a man-machine system, since the performance depends on both the system accuracy and the surgeon's perceptual ability. The evaluations showed the system to be highly effective in providing linear alignment assistance.
\end{abstract}

\section{Introduction}

Most surgical navigation systems display the graphical images used for navigation on a computer monitor positioned adjacent to the surgical scene, and

T. Dohi and R. Kikinis (Eds.): MICCAI 2002, LNCS 2489, pp. 125 1322002.

(C) Springer-Verlag Berlin Heidelberg 2002 
operative procedures are performed using a hand-held pointer and instruments with tracking markers [1],[2], [3]. These systems leave the surgeon the mental task of combining two sources of spatial information; because the images for navigation are supplied on a computer monitor, the surgeon has to look away from the surgical scene to obtain navigational information. Several types of augmented reality (AR) systems have been developed to address this problem, including image overlay displays [4, [5], [6], stereo image injection into binocular operating glasses [7, and a simple laser pointer [8]. However, these systems still have some drawbacks. Image overlay displays and binoculars may be cumbersome for use in some power-demanding orthopaedic procedures like hammering and drilling. A laser pointer is easy for surgeons to follow, but it has to move extensively around the surgical scene in order to point in various directions. Here, we propose a novel laser guidance configuration that overcomes the problems inherent in previous AR systems.

In this paper, we describe the principle and evaluate the performance of a laser guidance system that uses dual laser beam shooters fixed to a stand, the aim of which is to achieve the alignment of linear surgical tools such as drills and wires. In the proposed system, the intersection of two laser planes generated by dual laser shooters defines the straight insertion path of a surgical tool. The two laser planes are directly projected onto the patient and surgical tool to provide guidance information. Our assumption is that a linear surgical tool has cylindrical shape, or that a cylindrical sleeve is attached to a wire so that their axes are aligned. Using the properties the two laser plane projections on the entry surface of the patient and the cylindrical surface of the tool or attached sleeve, we propose a two-step procedure for position and orientation alignment to the straight insertion path. The procedure for orientation alignment is based on the human ability to perceive parallel lines. We evaluate the performance as a man-machine system since it is dependent on both the accuracy of the system and the perceptual ability of the surgeon.

\section{System and Guidance Procedure}

\subsection{Dual Laser System}

Figure 1 shows the configuration of the dual laser system and its appearance. The laser guidance system consists of two laser beam shooters that are fixed on stands $100-150 \mathrm{~cm}$ apart. Each shoots a $0.25-\mathrm{mW}$ red $(635 \mathrm{~nm})$ laser beam with 1-mm diameter spot. Each beam oscillates within the range of angle $\alpha$ at 50 $\mathrm{Hz}$, resulting in a beam tract in space shaped like a fan (in our system, $\alpha=10$ degrees.) The beam tract defines a fan-shaped plane segment, which we call the "laser plane". The two laser planes intersect in a line that can be controlled in any direction by changing the angle and direction of the beam oscillation using a galvanometer.

Let $O_{L}$ be the origin of the reference frame of the laser beam shooter. Let $\mathbf{e}_{\mathbf{x}}$, $\mathbf{e}_{\mathbf{y}}$, and $\mathbf{e}_{\mathbf{z}}$ be the coordinate axes. We assume that $\mathbf{e}_{\mathbf{z}}$ is the reference direction 


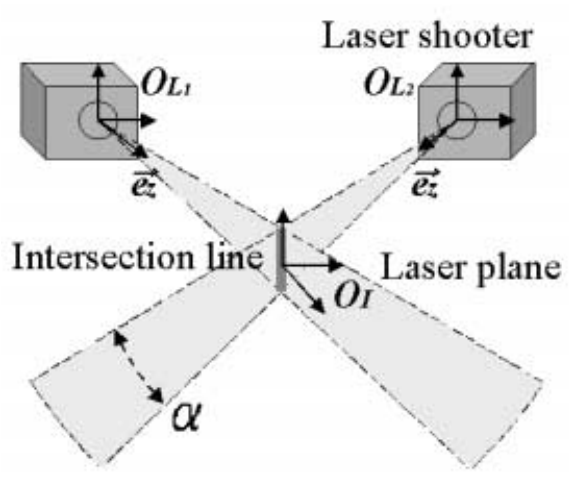

(a)

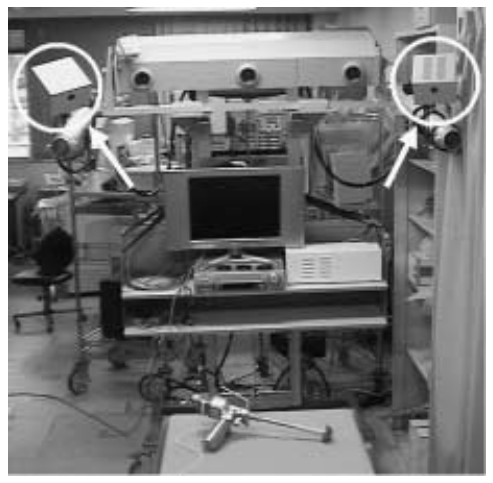

(b)

Fig. 1. Laser guidance system. (a) System configuration. (b) Appearance. Two laser beam shooters (shown by arrows) are attached to the OPTOTRAK camera.

of laser beam shooting. The laser plane is restricted to pass through $O_{L} 1$. The field of projection (FOP) of the laser plane of the laser shooter is roughly approximated by the cone-shaped volume whose apex, axis, and apex angle are $O_{L}, \mathbf{e}_{\mathbf{z}}$, and $\alpha$, respectively.

Let $\mathbf{n}=(\cos \phi \cos \theta, \cos \phi \sin \theta, \sin \phi)$ be the laser plane normal represented in the laser shooter frame. The possible parameter ranges of $\phi$ and $\theta$ are described as $-\frac{\beta}{2} \leq \phi \leq \frac{\beta}{2}$ and $-90^{\circ} \leq \theta \leq 90^{\circ}$, respectively (in our system, $\beta=10$ degrees.) The two laser shooters are arranged in the real $3 \mathrm{D}$ space so that their reference directions of laser beam shooting, $\mathbf{e}_{\mathbf{z}}$, roughly intersect. The approximated intersection point and the two origins of the reference frames form a triangle, the $z$-axes of the two shooters being projected to the intersection point. It is desired that this be an isosceles triangle satisfying $\left|O_{L_{1}}-O_{I}\right|=\left|O_{L_{2}}-O_{I}\right|=L$, where $O_{I}$ is the approximated intersection point and $O_{L_{1}}$ and $O_{L_{2}}$ are the origins of the two laser shooter reference frames, respectively. Using this configuration, a straight line of any direction passing through the spherical volume, whose center and diameter are respectively $O_{I}$ and $D=2 L \tan \frac{\beta}{2}$, can be specified by the intersection of the two laser planes. We used $L=150 \mathrm{~cm}$ as the length of the isosceles triangle. In this case, the diameter is approximately $D=2 \times 150 \times \tan 5^{\circ}=26 \mathrm{~cm}$.

\subsection{Guidance Procedure}

Given an insertion path described as a straight line, a laser plane that contains the given line and is generated by the laser shooter can be determined. Using the two generated planes whose intersection line is the insertion path, a guidance procedure is formulated below.

1 Precisely speaking, $O_{L}$ may deviate slightly from the laser plane depending on the mechanical properties of the galvanometer. This deviation is, however, practically negligible in terms of understanding the basic configuration of the system. In the actual system, we precisely calibrate the deviation to generate the laser plane. 
The guidance procedure consists of two steps (Fig.2). Firstly, the entry point is determined. The two laser planes are projected as crossing lines onto the entry surface. The tip of a surgical tool is then placed at the crossing point (Fig. 2(a)). Secondly, the orientation is determined after stabilization of the entry point. Two methods were considered for orientation guidance:

- Assuming that a coaxial point can be localized at the tail of the tool, the same method as that used for entry point guidance is employed.

- Assuming that a linear surgical tool has a cylindrical surface whose axis corresponds to the linear insertion path, projections of the laser planes onto the cylindrical surface of the tool are employed for the guidance.

We employed the latter method in the experiments described in this paper. The former method needs a relatively wide surface to receive the laser planes at the tail as well as a precisely localizable coaxial point, which is not easy to determine in a real situation. On the other hand, many linear surgical tools have a coaxial cylindrical surface. Also, in the case of wire insertion by drilling, it is easy to attach cylindrical guide sleeves to the wire. In general, a laser plane is projected as a quadratic curve onto a cylindrical surface. When the laser plane normal is orthogonal to the axis direction of the cylinder, however, the plane is projected as a straight line. Thus, the following property is derived:

- Two laser planes are projected as two parallel straight lines onto a cylindrical surface if and only if the direction of cylinder axis is aligned in the direction of the intersection line of the two laser planes.

Using this property, the orientation is adjusted while pivoting at the entry point so that the projections of the two laser planes onto the cylindrical surface are parallel and straight (Fig. 2(b)).

\section{Experiments}

\subsection{Calibration}

The proposed system was evaluated using an optical 3D position sensor (OPTOTRAK 3020; Northern Digital Inc., Waterloo, OT, Canada). The laser shooter reference frame was registered with the OPTOTRAK reference frame using the following method. Several laser beam lines (which do not oscillate), whose directions and positions are known in the laser shooter frame, are projected onto a plate at different positions. The projections of the laser beams are imaged as points, and their 3D positions digitized using an OPTOTRAK pen-probe. Assuming correspondences between the several laser beams and their projected points, the transformation matrix between the OPTOTRAK and laser shooter frames is determined.

\subsection{Method for Measuring Accuracy}

Given the insertion path as the intersection of the laser planes in the OPTOTRAK reference frame, the subject conducting the experiments aligned the entry 


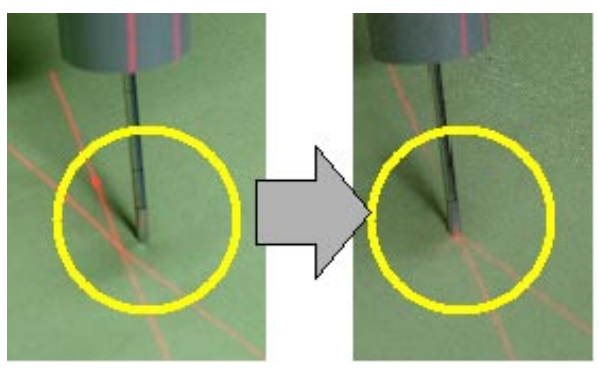

(a)

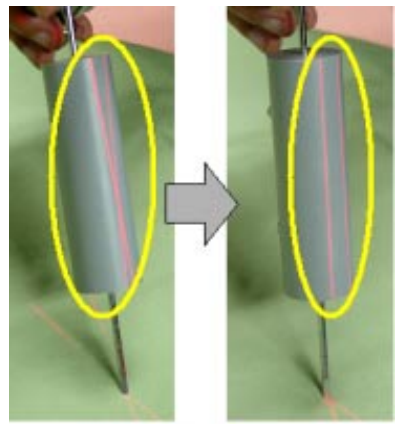

(b)

Fig. 2. Guidance procedure. (a) Positional alignment of entry point. (b) Orientation alignment of tool.

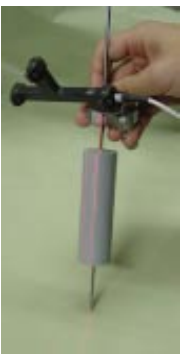

(a)

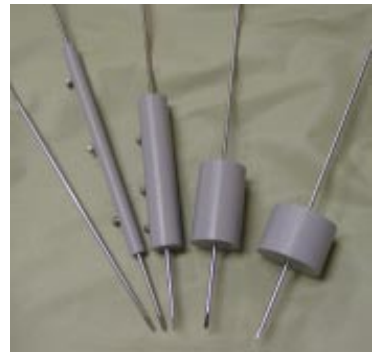

(b)

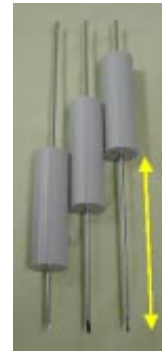

(c)

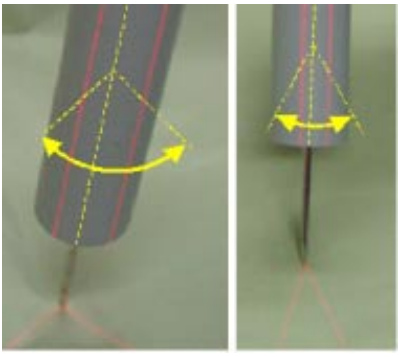

(d)

Fig. 3. Experimental materials for system evaluation. (a) Wire with cylindrical sleeve and AdapTrax. (b) Sleeves of different diameters and lengths. (c) Different sleeve positions. (d) Different intersecting angles of two laser planes.

point and direction of the cylindrical guide sleeve attached to the wire according to the guidance procedure described in section 2.2. The subject was an orthopaedic surgeon sufficiently trained to be able to perform the procedure appropriately. A tracking marker with LEDs (AdapTrax Tracker; Traxtal Technologies, Toronto) was fixed to the end of the wire. After calibration of the wire, the position of the entry point and direction of the wire were measured using an OPTOTRAK sensor (Fig. B(a)). Repeated measurements were made, and the measured positions and directions were compared with the specified positions and directions. The RMS error of the measurement system itself was confirmed to be about $0.5 \mathrm{~mm}$ in position and 0.5 degrees in direction.

\subsection{Method for Evaluating Effects of Diameter, Length, and Position of Cylindrical Sleeve}

To evaluate the effects of the diameter and length of the sleeve used to receive the laser beam on the accuracy of the wire insertion procedure and its usability by surgeons, twelve wire guide sleeves with different diameters and lengths were 
made (Fig. 3 (b)). The diameters were 3 (without sleeve), 10, 20, 30, and $40 \mathrm{~mm}$. The lengths were 50, 100, and $150 \mathrm{~mm}$. Gray was chosen for the sleeve color because the red laser beam was projected onto this color clearly. Kirshner wire $3 \mathrm{~mm}$ in diameter and $300 \mathrm{~mm}$ in length was used for the tests. To evaluate the effects of the guide sleeve position and the intersecting angle of the laser planes, sleeves were placed 50, 100, or $150 \mathrm{~mm}$ from the wire tip (Fig. 3. (c)) and intersecting angles of 10, 20, 30, 60, 90, 120, and 150 degrees were tested (Fig. 3(d)). The intersecting angle depends on both the direction of the specified insertion path and the arrangement of the two laser beam shooters. Measurements were repeated 20 times for each condition. Time spent by the subject on position alignment and orientation was also measured for each condition as an index of usability.

\subsection{Results}

Fig. 4 shows the experimental results on the angular accuracy of the orientation alignment and time spent on the alignment under various conditions. The effects of length of the guide sleeve, position of the sleeve, and the intersecting angle of the laser planes on angular accuracy are respectively shown in subfigures (a), (b), and (d) of Fig. 4 The effect of the sleeve diameter on the alignment time is shown in Fig. 4 (c).

The length and position of the sleeve had little effect on the angular accuracy of the wire direction (Fig. 4(a) and (b)). While the sleeve diameter also had little effect on the angular accuracy (result not shown), there was a tendency for the surgeon to need a longer time to align a wire with a smaller diameter sleeve (Fig. 4(c)). Considering both the accuracy of the wire direction and usability, the minimum sleeve length was judged to be $50 \mathrm{~mm}$ and the optimum sleeve diameter was in the range from 20 to $30 \mathrm{~mm}$. With respect to its position, the sleeve should be as distant from the wire tip as possible (Fig. 4(b)). When the sleeve was set within the above ranges, the accuracy of the wire direction was 0.6 degrees with 0.8 degrees of RMS. The intersecting angle of the laser plane affected the accuracy of the wire direction (Fig. 4(d)). The direction of the wire was most accurate when the angle was 60 degrees or 90 degrees, and these angles were the easiest for the surgeon to align the wire to the laser planes.

The positional accuracy of the wire tip placement was not much affected by the above conditions, being around $0.5 \mathrm{~mm}$ of bias with $0.9 \mathrm{~mm}$ of RMS (results not shown), except that the intersecting angle did have a small effect that was similar but weaker than the effect on the angular accuracy.

\section{Discussion and Conclusions}

The proposed laser guidance system with dual laser planes was shown to be capable of assisting surgeons in wire insertion with an accuracy of less than 1 $\mathrm{mm}$ for the wire tip position at the entry point and of less than 1 degree for the wire direction. The accuracy of the wire direction was not affected by the 


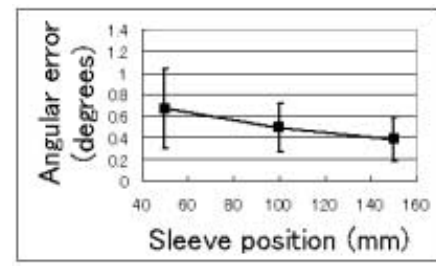

(a)

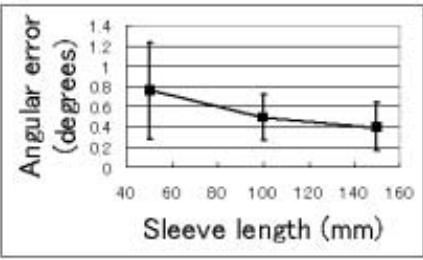

(b)

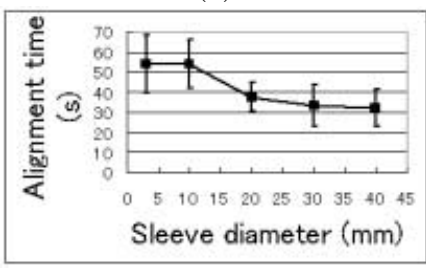

(c)

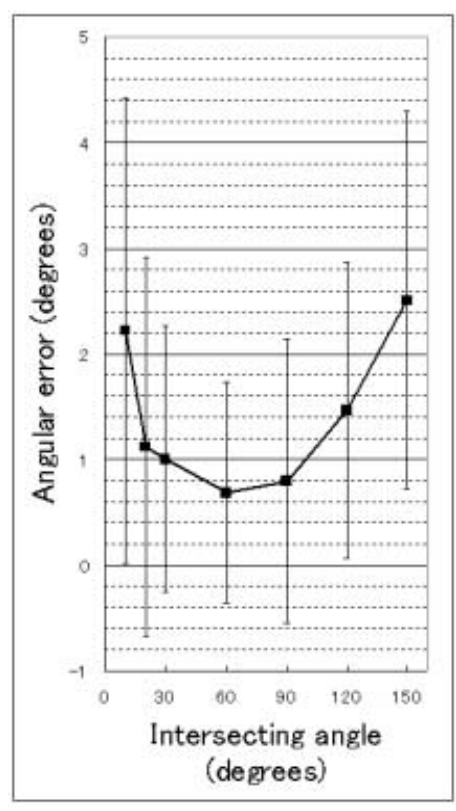

(d)

Fig. 4. Results of evaluations (a) Effect of length of guide sleeve on angular accuracy of orientation alignment. (b) Effect of position of guide sleeve on angular accuracy. (c) Effect of diameter of guide sleeve on alignment time. (d) Effect of intersecting angle of laser plane on angular accuracy.

length, position, or diameter of the sleeve, but it because easier for the surgeon to align the wire with the laser beam tracts as the length of the sleeve and its distance from the wire tip got shorter, and as the diameter of the sleeve became smaller. Considering the accuracy of the wire direction and the usability of the system by surgeons, the minimum sleeve length was judged to be $50 \mathrm{~mm}$, while the optimum sleeve diameter was in the range from 20 to $30 \mathrm{~mm}$. The optimum intersecting angle of the laser beam tracts was between 60 and 90 degrees.

The proposed system has the following advantages. The two laser shooters can be placed an fixed stands, while in a conventional laser system a laser pointer with a robotic arm is needed because it has to move extensively around the surgical scene to point in various directions [8]. Furthermore, since the laser beam direction is often close to surgeon's viewing direction, in the conventional system the beam can easily be occluded by the surgeon [8]. This does not happen in our system. Unlike in various other AR systems [4], [5], 6], [7], the proposed system does not require the wearing of glasses or mirrors to be placed between the surgeon and patient, although its function is limited to linear tool guidance. The surgeon who was the subject in our experiments reported that it was mentally quite easy to concentrate on aligning linear surgical tools since the guidance 
information was directly projected onto the surgical scene, allowing him to focus on it. In addition, he did not need to worry about occluding the laser beams.

It should be noted that the alignment performance was evaluated as a manmachine system. That is, the accuracy and alignment time depends on the overall performance of both the surgeon's perceptual ability (especially in judging whether or not two lines are parallel) and the accuracy of the system (including calibration and the mechanical accuracy of the galvanometer). The system effectively employs the keen ability of humans to judge parallel lines in achieving the alignment task, thereby requiring cooperation between human and machine. The overall accuracy and usability of this man-machine system was confirmed to be sufficiently high for clinical use.

We have already tested and reported on the clinical feasibility of the proposed guidance system [9], which was combined with our total hip replacement navigation system [2] and employed for guide wire insertion in acetabular cup placement. We confirmed that the system functioned successfully in the operating room [9].

A future problem to be addressed is guidance for the insertion depth. The current system does not provide guidance on how deep a tool should be inserted. We are now investigating methods for depth guidance, including the use of sound, blinking laser beams, and changing the laser beam color.

Acknowledgement: This work was partly supported by JSPS Research for the Future Program JSPS-RFTF99I00903.

\section{References}

1. Y Sato, M Nakamoto, Y Tamaki, et al., "Image Guidance of Breast Cancer Surgery using 3-D Ultrasound Images and Augmented reality visualization," IEEE Trans. Med. Imaging, vol.17, no.5, pp.681-693, 1998

2. Y Sato, T Sasama, N Sugano, et al., "Intraoperative Simulation and Planning Using a Combined Acetabular and Femoral (CAF) Navigation System for Total Hip Replacement," Lecture Notes in Computer Science, vol.1935 (MICCAI2000): pp.1114-1125, 2000.

3. A M DiGioia, B Jaramaz and B D Colgan, "Computer assisted orthopaedic surgery. Image guided and robotic assistive technologies," Clinical Orthopaedics and Related Research, No. 354, September, 1998.

4. M Blackwell, C Nikou, A M DiGioia, et al., "An Image Overlay System for Medical Data Visualization," Lecture Notes in Computer Science, vol.1496 (MICCAI'98): pp.232-240, 1998.

5. K Masamune, Y Masutani, S Nakajima, et al., Takeyoshi Dohi, Hiroshi Iseki and kinotomo Takakura, "Three-Dimensional Slice Image Overlay System with Accurate Depth Perception for Surgery," Lecture Notes in Computer Science, vol.1935 (MICCAI2000): pp.395-402, 2000.

6. S Nakajima, S Orita, K Masamune, et al., "Surgical Navigation System with Intuitive ThreeDimensional Display," Lecture Notes in Computer Science, vol.1935 (MICCAI2000): pp.403$411,2000$.

7. H Fuchs, A State, E D Pisano, et al., Gentaro Hirota, Mark A. Livingston, Mary C. Whitton, and Stephen M. Pizer, "Towards Performing Ultrasound-Guided Needle Biopsies from within a Head-Mounted Display", Lecture Notes in Computer Science, vol.1131 (VBC'96): pp.591-600, 1996

8. S Lavallée, J Troccaz, P Sautot, et al., "Computer-Assisted Spinal Surgery Using AnatomyBased Registration," in Russell H. Tayler, Stéphane Lavallée, Grigore C. Burdea, Ralph Mösges eds., "Computer-Integrated Surgery: Technology and Clinical Applications," The MIT Press, Cambridge, Massachusetts, pp.425-449, 1996.

9. N Sugano, T Sasama, S Nishihara, et al., "Clinical applications of a laser guidance system with dual laser beam rays as augmented reality of surgical navigation," Computer Assisted Radiology and Surgery, in press, 2002. 